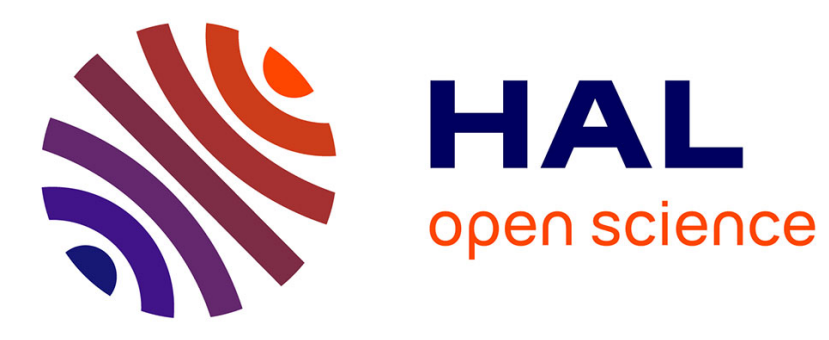

\title{
Targeting pheochromocytoma/paraganglioma with polyamine inhibitors
}

\author{
Sudhir Kumar Rai, Fernando Bril, Heather Hatch, Yiling Xu, Laura Shelton, \\ Srilaxmi Kalavalapalli, Arielle Click, Douglas Lee, Chris Beecher, Austin \\ Kirby, et al.
}

\section{To cite this version:}

Sudhir Kumar Rai, Fernando Bril, Heather Hatch, Yiling Xu, Laura Shelton, et al.. Targeting pheochromocytoma/paraganglioma with polyamine inhibitors. Metabolism, 2020, 110, pp.154297. 10.1016/j.metabol.2020.154297 . hal-02890700

\section{HAL Id: hal-02890700 https://hal.science/hal-02890700}

Submitted on 6 Jul 2020

HAL is a multi-disciplinary open access archive for the deposit and dissemination of scientific research documents, whether they are published or not. The documents may come from teaching and research institutions in France or abroad, or from public or private research centers.
L'archive ouverte pluridisciplinaire $\mathbf{H A L}$, est destinée au dépôt et à la diffusion de documents scientifiques de niveau recherche, publiés ou non, émanant des établissements d'enseignement et de recherche français ou étrangers, des laboratoires publics ou privés. 


\section{Journal Pre-proof}

Targeting Pheochromocytoma/Paraganglioma with polyamine inhibitors

Sudhir Kumar Rai, Fernando Bril, Heather M. Hatch, Yiling Xu, Laura Shelton, Srilaxmi Kalavalapalli, Arielle Click, Douglas Lee, Chris Beecher, Austin Kirby, Kimi Kong, Jose Trevino, Abhishek Jha, Shashank Jatav, Kriti Kriti, Soumya Luthra, Timothy J. Garrett, Joy Guingab-Cagmat, Daniel Plant, Prodip Bose, Kenneth Cusi, Robert A. Hromas, Arthur S. Tischler, James F. Powers, Priyanka Gupta, James Bibb, Felix Beuschlein, Mercedes Robledo, Bruna Calsina, Henri Timmers, David Taieb, Matthias Kroiss, Susan Richter, Katharina Langton, Graeme Eisenhofer, Raymond Bergeron, Karel Pacak, Sergei G. Tevosianc, Hans K. Ghayee

PII: S0026-0495(20)30161-X

DOI: https://doi.org/10.1016/j.metabol.2020.154297

Reference: YMETA 154297

To appear in:

Metabolism

Received date:

3 April 2020

Accepted date: 17 June 2020

Please cite this article as: S.K. Rai, F. Bril, H.M. Hatch, et al., Targeting Pheochromocytoma/Paraganglioma with polyamine inhibitors, Metabolism (2020), https://doi.org/10.1016/j.metabol.2020.154297

This is a PDF file of an article that has undergone enhancements after acceptance, such as the addition of a cover page and metadata, and formatting for readability, but it is not yet the definitive version of record. This version will undergo additional copyediting, typesetting and review before it is published in its final form, but we are providing this version to give early visibility of the article. Please note that, during the production process, errors may be discovered which could affect the content, and all legal disclaimers that apply to the journal pertain. 
(C) 2020 Published by Elsevier. 
${ }^{1}$ Sudhir Kumar Rai*, ${ }^{2}$ Fernando Bril*, ${ }^{3}$ Heather M. Hatch, ${ }^{1}$ Yiling Xu, ${ }^{4}$ Laura Shelton, ${ }^{1}$ Srilaxmi Kalavalapalli, ${ }^{5}$ Arielle Click, ${ }^{6}$ Douglas Lee, ${ }^{7}$ Chris Beecher, ${ }^{8}$ Austin Kirby, ${ }^{8}$ Kimi Kong, ${ }^{9}$ Jose Trevino, ${ }^{10}$ Abhishek Jha, ${ }^{10}$ Shashank Jatav, ${ }^{10}$ Kriti Kriti, ${ }^{10}$ Soumya Luthra, ${ }^{11}$ Timothy J. Garrett, ${ }^{11}$ Joy GuingabCagmat, ${ }^{12}$ Daniel Plant, ${ }^{12}$ Prodip Bose, ${ }^{2}$ Kenneth Cusi, ${ }^{8}$ Robert A. Hromas, ${ }^{13}$ Arthur S. Tischler, ${ }^{13}$ James F. Powers, ${ }^{14}$ Priyanka Gupta, ${ }^{14}$ James Bibb, ${ }^{15}$ Felix Beuschlein, ${ }^{16}$ Mercedes Robledo, ${ }^{16}$ Bruna Calsina, ${ }^{17}$ Henri Timmers, ${ }^{18}$ David Taieb, ${ }^{19}$ Matthias Kroiss, ${ }^{20}$ Susan Richter, ${ }^{21}$ Katharina Langton, ${ }^{20,}{ }^{21,}{ }^{22}$ Graeme Eisenhofer, ${ }^{23}$ Raymond Bergeron Jr., ${ }^{24}$ Karel Pacak, ${ }^{3}$ Sergei G. Tevosian, ${ }^{2}$ Hans K. Ghayee

*Authors contributed equally

${ }^{1}$ Department of Medicine, Division of Endocrinology, University of Florida, Gainesville, FL, USA

${ }^{2}$ Department of Medicine, Division of Endocrinology, University of Florida and Malcom Randall VA Medical Center, Gainesville, FL, USA

${ }^{3}$ Department of Physiological Sciences, University of Florida, Gainesville, FL, USA

${ }^{4}$ Scientific Project Development, Human Metabolome Technologies, Boston, MA, USA

${ }^{5}$ Department of Immunology, University of Texas Southwestern Medical Center, Dallas, TX, USA

${ }^{6}$ Omic Insight, LLC, Durham, NC, USA

${ }^{7}$ IROA Technologies, Chapel Hill, NC, USA

${ }^{8}$ Department of Medicine, University of Texas Health Science Center, San Antonio, TX, USA

${ }^{9}$ Department of Surgery, University of Florida, Gainesville, FL, USA

${ }^{10}$ Elucidata, Cambridge MA, USA

${ }^{11}$ Department of Pathology, Immunology, and Laboratory Medicine, University of Florida, Gainesville, FL, USA

${ }^{12}$ Department of Physiological Sciences, University of Florida, Malcom Randall VA Medical Center, Gainesville, FL, USA 
${ }^{14}$ Department of Surgery, University of Alabama, Birmingham, AL, USA

${ }^{15}$ Klinik für Endokrinologie, Diabetologie und Klinische Ernährung, UniversitätsSpital Zurich, Zurich, Switzerland

${ }^{16}$ Hereditary Endocrine Cancer Group, Spanish National Cancer Research Center (CNIO), and Centro de Investigación Biomédica en Red de Enfermedades Raras (CIBERER), Madrid, Spain

${ }^{17}$ Department of Internal Medicine, Radboud University Medical Center, Nijmegen, Netherlands

${ }^{18}$ Department of Nuclear Medicine, La Timone University Hospital, European Center for Research in Medical Imaging, Aix Marseille Université, Marseille, France

${ }^{19}$ Department of Internal Medicine, Division of Endocrinology and Diabetology, University Hospital Würzburg, University of Würzburg, Würzburg, Germany

${ }^{20}$ Institute of Clinical Chemistry and Laboratory Medicine, University Hospital Carl Gustav Carus, Technische Universität Dresden, Germany

${ }^{21}$ Department of Medicine III, University Hospital Carl Gustav Carus, Technische Universität Dresden, Germany

${ }^{21}$ Chief, Division of Clinical Neurochemistry, Institute of Clinical Chemistry and Laboratory Medicine, and Department of Medicine, Faculty of Medicine, University Hospital Carl Gustav Carus, Technische Universität Dresden, Germany

${ }^{23}$ Department of Medicinal Chemistry, University of Florida, Gainesville, FL, USA

${ }^{24}$ Eunice Kennedy Shriver National Institute of Child Health and Human Development, National Institutes of Health, Bethesda, MD, USA

Address Correspondence:

Hans K. Ghayee, DO

Division of Endocrinology 
L

Gainesville, FL 32610

hans.ghayee@medicine.ufl.edu

Sergei G. Tevosian, PhD

Department of Physiological Sciences

University of Florida

Gainesville, FL 32610

stevosian@ufl.edu

\begin{abstract}
Background: Pheochromocytomas (PCCs) and paragangliomas (PGLs) are neuroendocrine tumors that are mostly benign. Metastatic disease does occur in about $10 \%$ of cases, and for these patients no effective therapies are available. Patients with mutations in the succinate dehydrogenase subunit B (SDHB) gene tend to have metastatic disease. We hypothesized that a down-regulation in the active succinate dehydrogenase B subunit should result in notable changes in cellular metabolic profile and could present a vulnerability point for successful pharmacological targeting.
\end{abstract}


hPheo1 (hPheo1 $S D H B$ KD) cells. Additional analysis of 115 human fresh frozen samples was conducted. In vitro studies using $N^{1}, N^{11}$-diethylnorspermine (DENSPM) and $N^{1}, N^{12}$ - diethylspermine (DESPM) treatments were carried out. DENSPM efficacy was assessed in human cell line derived mouse xenografts.

Results: Components of the polyamine pathway were elevated in hPheo1 SDHB KD cells compared to wild-type cells. A similar observation was noted in SDHx PCC/PGLs tissues compared to their non-mutated counterparts. Specifically, spermidine, and spermine were significantly elevated in SDHx-mutated PCC/PGLs, with a similar trend in hPheo1 SDHB KD cells. Polyamine pathway inhibitors DENSPM and DESPM effectively inhibited growth of hPheo1 cells in vitro as well in mouse xenografts.

Conclusions: This study demonstrates overactive polyamine pathway in PCC/PGL with SDHB mutations. Treatment with polyamine pathway inhibitors significantly inhibited hPheol cell growth and led to growth suppression in xenograft mice treated with DENSPM. These studies strongly implicate the polyamine pathway in PCC/PGL pathophysiology and provide new foundation for exploring the role for polyamine analogue inhibitors in treating metastatic PCC/PGL.

Keywords: polyamine, pheochromocytoma (PCC), paraganglioma (PGL), SDHB, DENSPM, DESPM.

\section{Précis}

Cell line metabolomics on hPheo1 cells and PCC/PGL tumor tissue indicate that the polyamine pathway is activated. Polyamine inhibitors in vitro and in vivo demonstrate that polyamine inhibitors are promising for PCC/PGL treatment. 
Pheochromocytomas (PCC) and paragangliomas (PGL) are catecholamine-producing tumors derived from chromaffin cells of the adrenal medulla and sympathetic/parasympathetic chain ganglia, respectively. Patients afflicted with metastatic PCC/PGLs have a less than $40 \% 5$ year survival rate [1-3]. Hypertension and palpitations are the usual presenting symptoms, and devastating complications of excess catecholamine levels including cardiovascular collapse have been documented [4]. Surgical removal is the only definitive treatment, as reliable medical therapies to control tumor growth or metastatic lesions are limited $[5,6]$.

Despite major advances in understanding the genetic landscape of hereditary PCC/PGL syndromes, the biochemical mechanisms regulating growth and metastasis in these neuroendocrine tumors remain obscure and druggable targets have yet to emerge. The Cancer Genome Atlas (TCGA) consortium published the most comprehensive study of PCC/PGLs through multimodal genomic profiling in the largest assortment of tumors collected internationally, with standardized quality control and internally reviewed histopathology [7]. This work solidified a classification of PCC/PGL that includes independent tumor groups based on their profiling. Cluster 1 includes the hypoxia pathway associated tumors (with mutations in $V H L$ and $S D H x$ ). Cluster 2 includes tumors associated with kinase signaling pathway (with mutations in RET, NF-1, and TMEM127). Cluster 3 is composed of tumors carrying the Mastermind-like 3 (MAML-3) mutations [7-11].

The gene most closely associated with metastatic tumor behavior is the succinate dehydrogenase complex iron sulfur subunit B gene $(S D H B)$ that belongs to Cluster I [12-14]. SDHB is a subunit of a key Krebs cycle enzyme, succinate dehydrogenase, that is involved in conversion of succinate to fumarate [15, 16]. Mutations in the $S D H x$ genes are associated with accumulation of various intermediate metabolites controlling tumorigenesis, including excess succinate that is referred to as "oncometabolite" [17]. Using genetically engineered mouse cells, investigators have established that loss of $S D H B$ promotes cell proliferation via pyruvate carboxylation, succinate-induced hypoxia-inducible factor alpha (HIF $\alpha)$ stabilization, and enhanced gene methylation [18-20]. Data from these studies has provided valuable insight 
PCC/PGL[7] provided a unique opportunity for a comprehensive understanding of this rare neuroendocrine tumor by complementing it with metabolomic analysis of the same, extensively documented, tumor sets.

Several studies have indicated that neuroblastomas exhibit closely associated behavior with PCC/PGL, and recent work has shown that the polyamine pathway plays a key role in neuroblastoma pathogenesis. Polyamines (putrescine, spermidine and spermine) are ubiquitous polycations that bind nucleic acids and proteins and play essential roles in many fundamental processes ensuring normal cell growth and viability, including protein and nucleic acid metabolism, chromatin organization, ion channel control, protection from environmental assault damage and cell death regulation. Polyamines are required to maintain cell proliferation, and interplay between overcharged polyamine metabolism and several cancer pathways holds notable promise for drug therapy (reviewed in [21, 22]). The data on polyamine pathway in the adrenal medulla is scarce $[23,24]$.

While the role of polyamine pathway has not been previously considered for PCC/PGL, the possible link between neuroblastomas and the polyamine pathway was recognized thirty years ago [25]. Neuroblastoma, together with PCC and PGL, constitute the three known types of primary tumors that arise from the autonomic nervous system [26, 27]. Several genetic alterations have been described for neuroblastoma, most prominently amplification of $M Y C N$ proto-oncogene that predicts poor outcome [28, 29].. With respect to polyamine metabolism, ornithine decarboxylase (ODC, encoded by the ODC1 gene) is the key rate-limiting enzyme in polyamine synthesis pathway that converts ornithine to putrescine; its expression and function are tightly regulated at multiple levels [29]. ODC1 was one of the first direct MYC targets discovered and these genes are frequently co-amplified [30, 31]. Importantly, even in the absence of MYCN amplification, $O D C 1$ is overexpressed in high-risk compared to lower-risk tumors, and this overexpression is associated with reduced survival [32-34]. These earlier observations and several recent breakthroughs in understanding the role of the polyamine pathway in neuroblastoma $[35,36]$ suggest that over-active polyamine pathway could be an important negative factor in all primary autonomic nervous system tumors. 
proposed (reviewed in [37]). Importantly, homeostatic cellular polyamine concentrations are maintained through a finely orchestrated balance of synthesis and degradation as well as uptake and export, and targeting polyamine pathway for therapeutic purposes has to take these multiple processes into consideration. In this respect, down-regulation of ODC activity by its inhibitor difluoromethylornithine (DFMO) triggers compensatory increase in polyamine uptake $[38,39]$. While the mechanisms of polyamine transport are not entirely understood, synthetic polyamine analogues were developed that resemble natural polyamines, but do not support cellular growth [40]. These compounds could successfully reduce cellular polyamine concentrations while simultaneously jamming their import from the extracellular milieu.

Since succinate dehydrogenase mutations in PCC/PGL are associated with aggressive disease, we hypothesized that a down-regulation in the active succinate dehydrogenase B subunit should result in notable changes in cellular metabolic profile and could present a vulnerability point for successful pharmacological targeting. To examine the importance of the polyamine pathway in PCC/PGLs, we performed metabolite analysis on the progenitor human cell line originated from a PCC, hPheo1 wild-type (WT) [41] and its derivative, hPheo1 SDHB knock-down (KD) cells. We found that the polyamine pathway was up-regulated in hPheo1 SDHB KD cells and confirmed these results with PCC/PGL tissue samples with SDHx mutations.

\section{Materials and Methods}

\section{Cell culture:}

The hPheo1 WT cell line derived from a human progenitor PCC was previously described [41]. hPheo1 WT cells and hPheo1 SDHB KD cells were cultured in RPMI 1640 with $10 \%$ FBS. The BJ fibroblast cell line was cultured in DMEM with 10\% FBS. 
A six well plate (60 mm dish size) of hPheo1 WT cells and hPheo $1 S D H B$ KD cells $\left(3 \times 10^{6}\right)$ were grown in RPMI with $10 \%$ FBS and used for the extraction of intracellular metabolites after 72 hrs. Media was aspirated and cells were washed twice with $5 \%$ mannitol solution $(10 \mathrm{~mL}$ first and then $2 \mathrm{~mL}) .800 \mu \mathrm{L}$ of Optima LC/MS grade methanol (Fisher Scientific, Fairlawn, NJ, USA) was added to cells for 30 seconds to inactivate enzymes. Next, the cell extract was treated with $550 \mu \mathrm{L}$ of internal standards solution provided by Human Metabolome Technology, Inc., Tsuruoka, Japan (H3304-1002, Human Metabolome Technologies, Inc., Tsuruoka, Japan) and left undisturbed for another 30 seconds. The extract was obtained and centrifuged $2,300 \times \mathrm{g}$ at $4^{\circ} \mathrm{C}$ for $5 \mathrm{~min} .800 \mu \mathrm{L}$ of upper aqueous layer was centrifugally filtered through a Millipore 5-kDa cutoff filter at $9,100 \times \mathrm{g}$ and $4^{\circ} \mathrm{C}$ for $5 \mathrm{hr}$ to remove proteins. The filtrate was centrifugally concentrated and re-suspended in $50 \mu \mathrm{L}$ of Milli-Q water for CE-TOF/MS analysis.

\section{$S D H B$ knockdown, transduction and validation}

A total of $2 \times 10^{5}$ hPheo1 WT cells were seeded in 24 well plates and 750ul of SMARTvector Lentiviral Human SDHB shRNA (Dharmacon) were transfected using Polybrene Infection/Transfection Reagent (EMD Millipore, USA) and incubated overnight. Post-24 hrs, cells were rescued with ACL-4 media containing serum. At $48 \mathrm{hrs}$ post-transfection, cells were selected via addition of $500 \mu \mathrm{g} / \mathrm{ml}$ of puromycin for several weeks. RNA from puromycin-resistant cells was isolated and assayed by qRT-PCR.

\section{Cell line metabolomic analysis}

The metabolites were analyzed by using a fused silica capillary $(50 \mu \mathrm{m}$ i.d. $\times 80 \mathrm{~cm}$ total length), with commercial electrophoresis buffer (Solution ID:H3301-1001 for cation analysis (Solution ID:H3302-1021 for anion analysis), both from Human Metabolome Technologies) as the electrolyte. The sample was injected at a pressure of $50 \mathrm{mbar}$ for $10 \mathrm{sec}$ (approximately $10 \mathrm{~nL}$ ) in cation analysis and $25 \mathrm{sec}$ 
conditions were as previously described $[43,44]$.

Peaks were extracted using automatic integration software MasterHands (Keio University, Tsuruoka, Japan) to obtain peak information including $\mathrm{m} / \mathrm{z}$ and migration time for CE-TOF/MS measurement (MT), and peak area [45]. Signal peaks corresponding to isotopomers, adduct ions, and other product ions of known metabolites were excluded, and remaining peaks were annotated with putative metabolites from the HMT metabolite database based on their MTs and $m / z$ values determined by TOF/MS (Supplemental Table 1). The tolerance range for peak annotation was configured at $\pm 0.5 \mathrm{~min}$ for MT and $\pm 10 \mathrm{ppm}$ for $\mathrm{m} / z$. In addition, peak areas were normalized against those of internal standards and then the resultant relative area values were further normalized by sample amount using equation $1(\mathrm{Eq}-1)$.

$$
\text { Relative Peak Area }=
$$
Metabolite Peak Area

Metabolome measurements were carried out in a facility service at Human Metabolome Technology Inc. (Tsuruoka, Japan).

\section{Human Tissue:}

\section{PCC/PGL SDHB-deficient tumor samples}

Fresh frozen PCCs/PGLs were collected under protocols that also allowed for collection of ENSAT registry and biobank protocols as previously described [46]. PCC/PGL tumors were obtained from University Hospital Carl Gustav Carus, Technische Universität Dresden, Germany; NIH, USA; UniversitätsSpital Zurich, Switzerland; Tufts Medical Center, USA; Spanish National Cancer Research Center (CNIO), Spain; Radboud University Medical Center, Netherlands; Aix Marseille Université, France; and University of Würzburg, Germany. Ten normal adrenal tissue specimens were from the University of Alabama, USA. Tumor procurement was approved under Institutional Review Boards from each respective center/institution and it was used for validation of identified metabolites from hPheo1 SDHB KD and hPheo1 WT cell lines. 


\section{Journal Pre-proof}

\section{(A) Human tumor sample preparation}

Adrenal tumor tissue ( $\sim 5$ to $10 \mathrm{mg}$ ) was homogenized in $5 \mathrm{mM}$ ammonium acetate at $1800 \mathrm{rpm}$ for $30 \mathrm{sec}$. After 30 min incubation on ice, supernatant was collected by centrifuging at $20,000 \mathrm{xg}$ for 10 min at $4^{\circ} \mathrm{C}$. This supernatant was normalized to the amount of protein in the samples. Protein concentration was determined using Qubit protein quantification kit (Thermo Fisher, San Jose, CA). Following addition of 10 $\mu \mathrm{L}$ of internal standard solution, metabolites were extracted by adding 8:1:1 Acetonitrile : Methanol : Acetone $(\mathrm{v} / \mathrm{v})$ and incubating for $30 \mathrm{~min}$ at $4^{\circ} \mathrm{C}$. The internal standard solution consisted of D-Leucine$\mathrm{D}_{10}(\mathrm{C} / \mathrm{D} / \mathrm{N}$ Isotopes, Pointe-Claire, Quebec, Canada), L-Tryptophan-2,3,3-D $(\mathrm{C} / \mathrm{D} / \mathrm{N}$ Isotopes,), Succinic Acid-2,2,3,3- $\mathrm{d}_{4}\left(\mathrm{C} / \mathrm{D} / \mathrm{N}\right.$ Isotopes), L-Tyrosine Ring $-{ }^{13} \mathrm{C}_{6}$ (Cambridge Isotope Laboratories, Tewksbury, MA, USA), L-Leucine- ${ }^{13} \mathrm{C}_{6}$ (Cambridge Isotopes Laboratories), L-Phenylalanine Ring- ${ }^{13} \mathrm{C}_{6}$ (Cambridge Isotope Laboratories), N-BOC-L-tert-Leucine (Acros Organics, Fairlawn, NJ, USA), and N-BOC-L-Aspartic Acid (Acros Organics). Supernatant was dried under a gentle stream of nitrogen at $30^{\circ} \mathrm{C} .50 \mu \mathrm{L}$ of reconstitution solution consisted of BOC-L-Tyrosine, BOC-L-Tryptophan and BOC-D-Phenylalanine was added and samples were analyzed by LC-HRMS [47].

\section{Metabolite analysis by LC-HRMS:}

LC-HRMS metabolite analysis was conducted at the Southeast Center for Integrated Metabolomics (SECIM) at the University of Florida. Analysis was performed on a Thermo Q-Exactive Orbitrap mass spectrometer with Dionex UHPLC and autosampler. Samples were analyzed in positive and negative heated electrospray ionization (mass resolution of 35,000 at $\mathrm{m} / \mathrm{z} 200$ ) using separate injections (volumes of $2 \mu 1$ for positive and $4 \mu 1$ for negative were injected). Separation was achieved on an ACE 18 -PFP 100 x $2.1 \mathrm{~mm}, 2$ $\mu \mathrm{m}$ column with $0.1 \%$ formic acid in water as mobile phase $\mathrm{A}$ and acetonitrile as mobile phase $\mathrm{B}$. Flow rate was maintained at $350 \mu \mathrm{L} / \mathrm{min}$ with a column temperature of $25^{\circ} \mathrm{C}$ [47]. An internally curated metabolite library of 1100 compounds was used for metabolite identification by matching retention time $( \pm 0.2 \mathrm{~min})$ 
human PCC/PGL tumor samples carrying SDHx mutations, 74 non-SDHx mutated tumor samples, along with 10 normal adrenal medullae as controls) were profiled (Supplemental Table 2). Metabolite descriptions and pathways were derived using the Kyoto Encyclopedia of Genes and Genomes (KEGG) Database and the Human Metabolome Database (HMDB).

\section{Tumor data:}

Analysis of raw tumor metabolite data was performed on El-Maven as previously described (https://doi.org/10.5281/zenodo.2537593) [48]. Thermo .raw format files were converted to an open source format (mzML) using the msConvert tool from ProteoWizard [49]. Compound database used for processing was derived from KEGG database, with final compound database inclusive of all the compounds found in all of the KEGG's reaction databases. Automated peak picking feature of El-Maven was then used to get the peak intensity of all the metabolites. Polyamine metabolites were observed in positive mode. Cyclic Loess normalization [50] was performed followed by normalization with internal and injection standards to correct for any sample preparation biases or analytical errors. Batch effect correction was performed using edgeR package in $\mathrm{R}$ to correct for batch differences [51]. Differential expression analysis was performed using limma package in R to identify metabolites differentially enriched in different cohorts of interest [52].

\section{Network Analysis}

Network analysis was performed on Polly IntOmix [53]. Polly IntOmics is an integrated omics analysis tool based on GAM [54] and used to generate pathway level insights from metabolomics data. The network map was generated through Cytoscape and Inkscape tools [55]. See Supplemental Figure 1 for Cytoscape analysis.

\section{In-vitro drug testing by clonogenic assay}


per well in six well plates. After $24 \mathrm{hrs,} \mathrm{these} \mathrm{plates} \mathrm{were} \mathrm{treated} \mathrm{with} \mathrm{DENSPM} \mathrm{and} \mathrm{DESPM}$ concentrations between $10-75 \mathrm{nM}$ for 10 days [56]. Human BJ fibroblast cells were used as controls in colony forming assays.

After 10 days, the cells were stained with filter sterilized Crystal Violet $(0.1 \%, 25 \mathrm{~mL}$ methanol cell culture grade, $75 \mathrm{~mL}$ sterilized water) solution, $1.0 \mathrm{ml} /$ well for $30 \mathrm{~min}$. Plates were washed with distilled water and air dried overnight at room temperature. Then, they were scanned and colony frequency was measured and normalized with Image J (NIH, Bethesda, MD, USA) software version 1.4.

\section{Spheroid protocol for injections}

Cells were seeded at $5 \times 10^{4} / \mathrm{mL}$ in $7 \mathrm{~mL}$ of DMEM/F12 medium containing $2 \%$ B27, $1 \%$ penicillin/streptomycin, EGF $(20 \mathrm{ng} / \mathrm{mL})$ and bFGF $(10 \mathrm{ng} / \mathrm{mL})$ in ultra-low attachment $\mathrm{T} 25$ flasks (Corning). After a week, cells were passaged into $15 \mathrm{ml}$ of the medium at a density of $5 \times 10^{4} / \mathrm{ml}$ into T75 ultra-low flask and incubated for another week. Cell strainer was used to eliminate single cells. Cell clusters were washed with the collected medium, and the clusters were spun down at $900 \times \mathrm{g}$ for $10 \mathrm{~min}$ at $4^{\circ} \mathrm{C} .1 .5$ x $10^{6}$ accutase (ThermoFisher) was added to the cell pellet and incubated for $10-30$ min at $37^{\circ} \mathrm{C}$ in $\mathrm{CO}_{2}$ incubator. Cells were gently pipetted during the incubation several times. After dissociation, cells were spun down and re-suspended in DMEM/F12 medium. Cells were counted and 7.5 $\times 10^{5}$ cells/mouse injection were seeded and incubated for a week. The cell suspension containing spheres was transferred into a $50 \mathrm{ml}$ tube and spun down at $900 \times \mathrm{g}$ for $10 \mathrm{~min}$. Supernatant was discarded and the sphere pellet re-suspended in PBS, $100 \mu \mathrm{L} /$ mouse injection. Spheroids were kept on ice until the injection.

\section{Animals}

Commercial athymic scid male mice (Taconic C.B-Igh-1b/IcrTac-Prkdcscid) were used to generate a xenograft model of PCC/PGL. Animals were obtained from the vendor at 5 weeks and allowed to acclimate for two additional weeks. Subcutaneous tumors using hPheo1 WT and hPheo1-SDHB KD cells were created 
subcutaneously into the right flank of animals. The control cohort was injected with PBS only. Animals were monitored biweekly until tumors started to appear (were palpable) at approximately two months, at which point animals were monitored daily. When the tumors reached a well-defined, caliper-measurable size (100-200 mm3), animals carrying either hPheo1 WT or hPheo1 SDHB KD xenografts were inspected by the MRI and divided into two groups, the PBS-injected (control group) or DENSPM-injected (treated group). The protocol to examine the effects of DENSPM in xenograft animals was approved by the UF IACUC (Study \#201609547). Once the tumor in the untreated animals reached the predetermined size of $\sim 2000 \mathrm{~mm} 3$, they were inspected by the MRI, sacrificed and tumor tissue preserved. Furthermore, MRI was used to detect tumors and measure tumor growth by volumetric assessment at the completion of the experiment. Between MRI procedures, tumors were measured every other day using digital caliper and tumor volume $(\mathrm{V})$ was calculated using the formula $\mathrm{V}=\mathrm{ab}^{2} \times 0.52$, where $\mathrm{a}$ and $\mathrm{b}$ are major and minor axes of the tumor foci, respectively. hPheo1 WT and hPheo1-SDHB KD-injected mice with tumor volume at $\sim 100 \mathrm{~mm}^{3}$ were divided into two following treatment groups with similar tumor volume distribution: (1) vehicle or (2) DENSPM administered by intraperitoneal injection at $60 \mathrm{mg} / \mathrm{kg}$ QD. The concentration of the DENSPM was based on previous work [59]. Bernacki et al. [59] observed some toxicity and weight loss at a dose of $80 \mathrm{mg} / \mathrm{kg}$ so here a lower dose $(60 \mathrm{mg} / \mathrm{kg})$ and lower frequency of administration (QD vs. TID) was used. All treated animals were kept on treatment for 46 days, at which point they were inspected by the MRI, sacrificed and tumor tissue (if any) preserved. Animal weight and normal activity were monitored.

\section{MRI Procedures}

Mice were imaged using a MR Solutions quadrature birdcage in a mouse cradle (Minerve Equipement Veterinaire, Esternay, France) maintained at $37^{\circ} \mathrm{C}$. Hearing protection and eye lubricant were applied and the animals anesthetized with $2 \%$ to $2.5 \%$ isoflurane at $0.8 \mathrm{~L} / \mathrm{min}$ oxygen flow rate. Respiration was monitored (SA Instruments Inc., Stony Brook, NY) using a respiration pillow sensor under the abdomen. Waste gas and $\mathrm{CO}_{2}$ were actively scavenged. Total scan time including localizer acquisitions was 30-45 min 
high-resolution MRI data was collected on a 7T MRI system (MR Solutions Ltd, Guildford, UK) equipped with a shielded gradient system with $300-\mathrm{mT} / \mathrm{m}$ gradient strength and a clear-bore diameter of $90 \mathrm{~mm}$. Scout localizer images were collected with a gradient-echo sequence followed by T1-weighted anatomical images (RARE;TE $=8 \mathrm{~ms}$ ) to confirm the slice package. This slice package was used to collect T2-weighted data for analysis. Twenty-four contiguous slices $1 \mathrm{~mm}$ thick were collected using RARE with standard CHESS Fat Suppression. Field of view was $32 \mathrm{~mm}$ x $32 \mathrm{~mm}$ and the matrix size was 400 x 256 which provided an in-plane resolution of $80 \mu \mathrm{m} \times 125 \mu \mathrm{m}$. Initial TE was $70 \mathrm{~ms}$ and the echo train length was 8 with echo spacing of $10 \mathrm{~ms}$. TR was 4 seconds and the number of acquisitions was equal to 4 resulting in a total acquisition time of 13.5 minutes. Mean tumor volumes were measured by drawing regions of interest (ROI), to circumscribe the entire tumor.

\section{Necropsy Analysis}

Prior to necropsy, the investigator was unaware of the results of the caliper measurements or imaging scans (that were done by different lab personnel), rendering the two analyses independent. $24 \mathrm{hr}$ after the last dose ( or when the untreated tumors reach $>2,000 \mathrm{~mm}^{3}$ ), animals were imaged by the MRI and euthanized. Mice were examined at the injection site, tumors excised and weighted. Tumor weights at this point were used as the endpoint reading. To measure the differences between the two tumor models and to gage DENSPM treatment efficacy, at least 6 animals for each group were measured with calipers on continuous days to obtain longitudinal data and the results were plotted.

\section{Total RNA isolation and quantitative Real Time (qRT)-PCR}

Total RNA were extracted from hPheo1 WT, and hPheol SDHB KD cells and by the Direct-Zol RNA Minipreparation plus Kit (Zymo Research,USA) following the manufacturer's protocol. $20.0 \mathrm{ng}$ of purified RNA were used for Quantitative real-time PCR using Luna ${ }^{\circledR}$ Universal Probe One-Step RT-qPCR Kit (NEB) and Step One Plus Real-time PCR system (Applied Biosystems) following the manufacturer's 
in Supplemental Table 3. SDHB gene expression levels were normalized to GAPDH and relative expression was calculated using the comparative threshold cycle method $\left(\Delta \Delta \mathrm{C}_{\mathrm{T}}\right)$ with the Stepone software (Applied Biosystems). Fold changes in gene expression were calculated with normalized value and represented in $\log _{2}$ value on Y-axis.

\section{Western blot}

Cells were lysed in $100 \mu \mathrm{L}$ of Pierce IP Lysis buffer with $1 \mathrm{X}$ final concentration of protease inhibitor (Product: 78430, Thermoscientific USA), $200 \mathrm{mM}$ EDTA and phosphatase inhibitor (Product: 78428, Thermoscientific USA) cocktail mixture solution for $30 \mathrm{~min}$ on ice and centrifuge at $14000 \times \mathrm{g}$ for $15 \mathrm{~min}$. Clear supernatant was collected in fresh tube and stored at $-80^{\circ} \mathrm{C}$ until further use. $60 \mu \mathrm{g}$ of whole cell protein extract of hPheo1 WT and hPheo1 $S D H B$ KD were separated by $4-20 \%$ sodium dodecyl sulfate polyacrylamide gel electrophoresis and transferred to PVDF membrane (Millipore, Bedford, MA). After blocking with 5\% dry milk in 1X TBST (Tris base pH7.4, KCl $2.7 \mathrm{mM}, \mathrm{NaCl} 137 \mathrm{mM}$ and 0.01\% Tween) for $2 \mathrm{hr}$ at room temperature, membranes were incubated with the primary antibodies against SDHB (1:2000), and eEF2 (1:2000) in dilution buffer overnight at $4^{\circ} \mathrm{C}$. Membranes were washed for three times with TBST and incubated with HRP conjugated mouse secondary antibody (NA931-1ML) and Rabbit IgG, HRP-linked Whole Ab (NA934-1ML) with 1:5000 dilution. PVDF blots were developed using hyBlot CL (Denville Scientific, Inc, USA) autoradiography film. Signal was normalized with eEF2 internal loading control and fold difference was measured by Image J software (NIH, Bethesda, USA).

Antibodies used were as follows: SDHB (Ab14714, Abcam) and eEF2 (\#2332, Cell Signal Technology), and Rabbit IgG, HRP-linked Whole Ab (NA934-1ML), HRP conjugated mouse secondary antibody (NA931-1ML) (Thermo Fisher Scientific, Waltham, MA).

\section{Statistical analysis}


normally-distributed variables and Kruskal-Wallis for non-parametric variables. Due to small sample size, cell line-based analyses were also performed with non-parametric tests. P values of $<0.05$ were considered statistically significant. Statistical analyses were performed with Stata 11.1 (StataCorp LP, College Station, TX) and Prism 6.0 (GraphPad Software, Inc., La Jolla, CA).

\section{Results:}

\section{hPheo1 SDHB KD cells:}

hPheo1 $S D H B \mathrm{KD}$ cells were generated as described in the Materials and Methods section and $S D H B$ expression was analyzed. Delta $\mathrm{Ct}$ value was normalized with $G A P D H$ and data represented in $\log _{2}$ value on Y-axis (Figure 1A). The qRT-PCR shows a decrease in $S D H B$ mRNA expression in hPheo1 SDHB KD compared to hPheo1 WT (Figure 1A). Since $S D H B$ RNA is reduced but not absent in hPheol cells, hPheo1 WT and hPheo1 $S D H B \mathrm{KD}$ cells were examined for the presence of SDHB protein expression using immunoblot (Figure 1B); ubiquitous (e.g., [60]) eukaryotic translation elongation factor 2, eEF2 was used as a loading control as it tracks well in these cells with other customarily used loading controls. As shown in Figure 1, hPheo1 SDHB KD showed significantly reduced levels of mRNA, and almost complete absence of SDHB protein expression.

\section{Polyamine metabolism}

To assess the metabolic changes in hPheo 1 cell line after $S D H B \mathrm{KD}$, succinate levels were measured, which were significantly higher in hPheo1 $S D H B \mathrm{KD}$ cells compared to hPheo1 WT cells (Figure 2). Analysis of 31 primary tumor samples with $S D H x$ mutations demonstrated a 20 -fold increase in succinate levels compared to non-SDHx-mutated adrenal tumor samples $(\mathrm{p}<0.001)$ (Figure 2), in line with previously reported data [61]. 
hPheo1 $S D H B \mathrm{KD}$ cells compared to WT cells. In order to confirm this data, we used SDHx mutated tumor tissue (SDHAF2 [n=1], SDHB [n=18], SDHC [n=3], and SDHD [n=9]) compared to non-SDHx mutated tumor samples. Particularly, significant increases for spermidine and spermine (Figure 2) were observed in human SDHx mutated PCC/PGLs, with a similar pattern in hPheo1 SDHB KD cells, although spermidine did not reach statistical significance in cell line experiments. On the other hand, putrescine (Figure 2) showed decreased levels in hPheo1 $S D H B$ KD cells, but not in human $S D H x$-mutated samples. Of note, in a sub-analysis comparing only $S D H B$ metastatic samples $(n=6)$ to normal adrenal medulla $(n=10)$, we observed an overall similar trend. Both spermidine and spermine were higher in metastatic samples with $S D H B$ mutation, although they did not reach statistical significance. However, ornithine and putrescine, which are upstream of spermidine and spermine in the polyamine pathway, did reach statistical significance when $S D H B$ malignant samples were compared to normal adrenal medulla ( $2.8 \pm 0.6$ vs. $1.0 \pm 0.3, \mathrm{p}=0.008$; $2.1 \pm 0.4$ vs. $1.0 \pm 0.2, \mathrm{p}=0.012$, respectively). Also, when comparing metastatic $S D H B$ samples $(\mathrm{n}=6)$ versus benign $S D H B$ samples $(\mathrm{n}=12)$ ornithine levels were statistically higher in metastatic samples compared with benign samples $(2.8 \pm 0.6$ vs. $1.6 \pm 0.2, \mathrm{p}=0.023$, respectively). In terms of functional tumors, evaluating $S D H x$ tumors making dopamine/norpepinephrine (n=93) compared to $S D H x$ tumors that were nonfunctional $(\mathrm{n}=8), S D H x$, functional samples had significantly lower putrescine $(1.5 \pm 0.1$ vs. $2.7 \pm$ $0.7, \mathrm{p}=0.009)$ and spermidine $(4.0 \pm 1.3$ vs. $15.7 \pm 5.3 \mathrm{p}=0.003)$. When specifically comparing $S D H B$ functional vs. $S D H B$ non-functional samples, a trend towards lower putrescine $(1.6 \pm 0.2$ vs. $3.1 \pm 2.0$, $\mathrm{p}=0.07)$ and spermidine $(2.7 \pm 1.1$ vs. $11.5 \pm 10.8, \mathrm{p}=0.06)$ in functional $S D H B$ was observed (Figure 2).

Overall, these results are consistent with polyamine pathway over-activity with increased production of spermidine and spermine, which is more significant in non-functional (i.e., less differentiated) tumor samples. While this is enough to produce a significant reduction of putrescine levels in hPheo1 $S D H B \mathrm{KD}$ cells, it is likely that sustained influx of substrate in human adrenal tumors is able to maintain the increased polyamine pathway activity without causing putrescine depletion. 
Differences in levels of polyamine pathway components in hPheo1 WT and hPheo1 SDHB KD cell lines prompted us to examine their response to polyamine analogue inhibitor (DENSPM and DESPM) treatment. All three cell lines (BJ fibroblasts, hPheo1WT and hPheol $S D H B \mathrm{KD}$ ) demonstrated different doubling times. BJ fibroblasts, hPheo1WT, and hPheo1 SDHB KD cells were cultured and treated with different doses of DENSPM ranging between 10nM-75nM with 500 cells/well (Figure 3A). DENSPM dose response curves showed $\mathrm{IC}_{50}$ value for hPheo1WT at $75 \mathrm{nM}$ whereas hPheo1 SDHB KD showed a response at 40nM (Figure 3B). On the other hand, BJ fibroblasts showed no sensitivity towards DENSPM at this concentration range (Figure 3B). For DESPM, the dose response data demonstrated a very low $\mathrm{IC}_{50}$ for hPheo $1 S D H B \mathrm{KD}$ at $10 \mathrm{nM}$, whereas hPheo1 WT showed an $\mathrm{IC}_{50}$ of 50nM (Figure $3 \mathrm{C}$ and D).

\section{Animal xenograft experiments}

The remarkable growth suppression of hPheo1 cell growth by DENSPM and DESPM encouraged us to investigate in vivo effect of polyamine inhibitors. DENSPM was chosen for in vivo studies due to its reported lower toxicity [62]. The antitumor effect of DENSPM was evaluated in the xenograft model as described in the Methods. No significant weight loss or signs of toxicity (i.e., diarrhea, abdominal stiffness, lethargy) were noted at that concentration in the treated group $(60 \mathrm{mg} / \mathrm{kg}$ DENSPM by intraperitoneal injection) compared to vehicle. Tumor size was measured with digital calipers and tumor volume was calculated (Figure 5A). DENSPM administration resulted in a reduction of tumor volume, with tumor growth inhibition (TGI) reaching $75 \%$ and $86 \%$ in hPheo1 and hPheo1 SDHB KD cohorts, respectively. DENSPM significantly reduced tumor volume relative to control between days 16 and 36, with a TGI of $\sim 66 \%$ in both cohorts by day 16 . Tumor weight in treated animals was also significantly reduced compared to vehicle-injected mice (Figure 5B). Quantitative assessment of tumor growth was additionally performed by MRI (Figure 5C). MRI confirmed DENSPM-mediated tumor regression (i.e., tumor being smaller by the end of the experiment) in 8 out of $10 \mathrm{hPheo} 1 \mathrm{SDHB} \mathrm{KD}$ mice with an overall $87 \%$ regression in tumor volume relative to the first MRI measurement ( 3 days after the beginning of treatment). 


\section{Discussion}

In order to identify new altered metabolic pathways due to $S D H x$ mutations, we conducted a metabolite analysis in hPheo1 WT and hPheo1 SDHB KD cells and confirmed our results in human PCC/PGLs tissue since there is only one progenitor human cell line derived from PCC in existence. As shown in Figure 2 of the present study, with $S D H B \mathrm{KD}$ in hPheo1 cells, there was an elevation in succinate compared to hPheo1WT. Moreover, this finding was further confirmed in experiments performed in human tumor tissue. These results are in accordance with prior reports [61, 63-66] suggesting that SDHx mutations lead to the accumulation of succinate, which in turn can act as oncometabolite, altering gene expression and epigenetic regulation and favoring PCC/PGL progression[17, 67].

Moreover, hPheo1 SDHB KD cells also showed increased polyamine pathway end products (i.e., spermidine and spermine), suggesting that this pathway may be up-regulated in these tumors. This trend was confirmed in PCC/PGL SDHx human tumors. Furthermore, polyamine substrate increases appeared to be more significant among non-functional tumors, suggesting that upregulation of this pathway may play a role in the survival of more undifferentiated tumors. These findings were consistent among all SDHx mutated samples, but also when the analyses were limited to $S D H B$ samples. The reasons for the elevation of the polyamines in cells with altered TCA cycle metabolism is currently under investigation. It has been demonstrated that in the adrenal gland, the polyamine, putrescine (and not glutamic acid), serves as a sole precursor for gamma-aminobutyric acid (GABA) synthesis [68]. Under certain circumstances, GABA can be converted to succinate and enter the tricarboxylic acid cycle (TCA), through the so-called "GABA shunt" pathway [69]. It is possible that excess succinate leads to the feedback inhibition of GABA synthesis and accumulation of polyamine products.

Recent advances highlighted the importance of the polyamine pathway in a type of tumor closely related to PCC/PGL, such as neuroblastoma. It was long known that autonomic nervous system tumors have many important features in common, and similar features between PCC/PGL and neuroblastoma have been reported (e.g., [70-72]). Recent research highlighted the role for solute carrier family 3 member 2 (SLC3A2) 
radiolabeled spermidine was reduced in cells with knockdown of SLC3A2 in neuroblastoma cells. Furthermore, inhibition of polyamine uptake combined with the suppression of the polyamine synthesis through ODC1 inhibition abrogated tumor development in neuroblastoma-prone mice [35]. Therefore, we hypothesized that as polyamine pathway appears to be up-regulated in hPheo1 SDHB KD cells, they would be sensitive to chemically synthesized polyamine pathway inhibitors $N^{l}, N^{l 1}$-diethylnorspermine (DENSPM) and $N^{1}, N^{12}$ - diethylspermine (DESPM) even at low concentrations, ranging from 10nM-100nM (see Figure 3). When the cell lines were treated with DESPM, hPheo1 SDHB KD cells were found to be more sensitive compared to hPheo1WT. However, both hPheol cell lines were more sensitive to DENSPM compared to human fibroblasts. Although a more dramatic effect was seen with DESPM treatment of hPheo1 $S D H B \mathrm{KD}$ cells compared to hPheo1 WT, we used DENSPM for in vivo experiments as DENSPM is currently being examined in human clinical trials in other cancers as it is less toxic than DESPM [56]. This is consistent with the observations of a number of different cancer tumor lines $[56,62]$.

Based on these findings, we tested polyamine pathway inhibitors to examine their effect on cellular proliferation in vitro and in mouse xenograft models. We also showed here that DENSPM significantly decreases tumor growth in vivo in our physiologically relevant, patient-derived cell line xenograft mouse model. Taken together, these results suggest that delivery of a well-tolerated dose of DENSPM will result in substantial activity against PCC/PGL derived tumors. Better understanding of the mechanistic basis of action for polyamine analogue compounds in PCC/PGL will inform a new therapeutic approach for patients with metastatic disease.

\section{Conclusion:}

Novel to the understanding of PCC/PGL pathophysiology there is a significant up-regulation of the polyamine pathway in hPheol $S D H B \mathrm{KD}$ cells as well as in human $S D H B$ tumor tissue. Furthermore, treatment with polyamine inhibitors significantly inhibited hPheol cell growth and led to growth inhibition in xenograft mice treated with DENSPM. These results provide new foundation for exploring the role for 
studies are needed to explore if this drug can be considered for clinical use in PCC/PGL patients.

Author Contributions: Idea formulation: H.K.G., G.E., S.T., R.B., R.A.H., and K.P.; methodology: S.K.R., F.B., Y.U. H.M.H., S.K. J.T.; analysis: F.B., S.K., L.S., A.J., K.K., S.J., S.L.; investigation: S.K.R., F.B., H.M.H., Y.U., L.S., S.K., A.C., C.B., D.L., D.P., P.B., A.K., J.C., T.J.G. resources: R.B., R.A.H., K.C., P.G., J.B., A.S.T., J.F.P., F.B., M.R., B.C., M.K., D.T., H.T., S.R., K.L., K.P., S.G.T., and G.E.; writing original manuscript draft: S.K.R., F.B., S.G.T., and H.K.G; project administration: H.K.G., S.G.T., and G.E.; funding acquisition: H.K.G.; K.C., K.K., R.A.H.

Acknowledgements: The authors would like to thank the patients, their families, and the administration of SDHB PheoParaCoalition for supporting this research. This work was also supported by the Gatorade Trust through funds distributed by the University of Florida, Department of Medicine, and Pilot Funding Initiative of the North Florida/ South Georgia (NF/SG) VA HS Research Service to HKG.

Funding: This research was supported by the Gatorade Trust Fund from the Department of Medicine, University of Florida Gatorade (Project\# 00122235), Southeast Center for Integrated MetabolomicsUniversity of Florida, and Malcom Randall VA Pilot Award Program (H.K.G); Eunice Kennedy Shriver National Institute of Child Health and Human Development, National Institutes of Health (K.P.). The sponsors had no involvement in study design, in the collection, analysis and interpretation of data. in the writing of the report and in the decision to submit the article for publication. 
development. H.K.G. received royalties from the University of Texas Southwestern Medical Center at Dallas.

\section{Figure Legends}

Figure 1: hPheo1 WT and hPheo1 SDHB KD cell lines. (a) Quantitative Real Time-PCR analysis in hPheo1 WT(ם) and hPheol SDHB KD (口) are shown; mRNA level were normalized with GAPDH. Results show mean $\pm \mathrm{SD}, \mathrm{n}=3$. (b) Total-cell extracts were prepared and analyzed by western blot for SDHB protein expression. Note decreased expression of SDHB in both experiments.

Figure 2: Polyamine pathway and altered succinate. Comparison of succinate level in hPheo1 WT and hPheo1 $S D H B \mathrm{KD}$ are represented on the left and PCC/PGL tumor tissues of non-SDHx mutated samples and SDHx mutated samples are compared on the right side. Y-axis represents relative intracellular metabolite levels respective to normal samples. Succinate levels are elevated in cells carrying $S D H x$ mutation and tumor tissue samples. Significant increases for spermidine and spermine were observed in human $S D H x$-mutated PCC/PGLs; a similar pattern was observed in hPheo1 SDHB KD cells, although spermidine did not reach statistical significance in cell line analysis.

Figure 3A-D: (A-B). Polyamine analog DENSPM was used for treatment of BJ fibroblasts (…), hPheo1 WT (---) and hPheo1 SDHB KD (-) cells at 10nM-75nM. (A) The quantitative analysis of the colonyforming assay is represented in left panel. (B) Representative images of colony forming assay for BJ fibroblasts, hPheo1 WT, and hPheo1 $S D H B \mathrm{KD}$ cells. All experiments were performed in triplicate and represented as mean \pm SD. (C-D). Polyamine analog DESPM was used for treatment of BJ fibroblasts ( $\cdots$ ), hPheo1 WT (---) and hPheo1 SDHB KD (-) cells at 10nM-50nM. (C) The quantitative analysis of the 
BJ fibroblast, hPheo1 WT, and hPheo1 $S D H B \mathrm{KD}$. All experiments were performed in triplicate and represented as mean $\pm \mathrm{SD}$. Note that DENSPM dose response curves showed $\mathrm{IC}_{50}$ value for $\mathrm{hPheo} 1 \mathrm{WT}$ at 75nM, whereas hPheo1 $S D H B \mathrm{KD}$ showed a response at 40nM (3B). Also note that BJ fibroblasts showed no sensitivity towards DENSPM at this concentration range (3B). For DESPM, the dose response data demonstrated a very low $\mathrm{IC}_{50}$ for $\mathrm{hPheo} 1 \mathrm{SDHB} \mathrm{KD}$ at $10 \mathrm{nM}$, whereas hPheo1 WT showed an $\mathrm{IC}_{50}$ of 50nM (3C and 3D).

Figure 4A-C. DENSPM treatment in hPheo1 WT and hPheo1 SDHB KD xenograft models. (A) Four cohorts of male mice (Taconic C.B-Igh-1b/IcrTac-Prkdcscid; $n=6-10$ per cohort) were administered vehicle control (PBS) or DENSPM $(60 \mathrm{mg} / \mathrm{kg})$ via i.p. injection, daily over 6 weeks to generate tumor growth curves of cell line xenografts. Tumor volumes are displayed as means \pm SD $(* * * P<0.001)$. (B) Weights of tumors isolated at endpoint after 45 days of treatment. Tumor weights are displayed as means \pm SD $(* * * P<0.001)$. (C) Representative three-dimensional images of the tumors obtained through MRI volumetric analysis in four animal cohorts as indicated, before and after treatment with either PBS or DENSPM. Note that DENSPM administration resulted in a reduction of tumor volume, with tumor growth inhibition (TGI) reaching $75 \%$ and $86 \%$ in hPheo1 WT and hPheo1 SDHB KD cohorts, respectively. DENSPM significantly reduced tumor volume, with a TGI of $\sim 66 \%$ in both cohorts by day 16 . Tumor weight in treated animals was also significantly reduced compared to vehicle-injected mice (4B).

Figure 5. Graphic summary of key results. Polyamine pathway is overactive in cells carrying $S D H B$ mutations and in PCC/PGL $S D H x$-mutated tumor tissue samples. Treatment with polyamine pathway inhibitors preferentially suppresses growth of hPheo1 SDHB KD and hPheo1 WT compared to control cells in culture and in a mouse xenograft model. 


\section{References:}

[1] Ziegler RG, Weinstein SJ, Fears TR. Nutritional and genetic inefficiencies in one-carbon metabolism and cervical cancer risk. J Nutr. 2002;132:2345S-9S doi: 10.1093/jn/132.8.2345S.

[2] Turkova H, Prodanov T, Maly M, Martucci V, Adams K, Widimsky J, Jr., et al. Characteristics and Outcomes of Metastatic Sdhb and Sporadic Pheochromocytoma/Paraganglioma: An National Institutes of Health Study. Endocr Pract. 2016;22:302-14 doi: 10.4158/EP15725.OR.

[3] Hamidi O. Metastatic pheochromocytoma and paraganglioma: recent advances in prognosis and management. Curr Opin Endocrinol Diabetes Obes. 2019;26:146-54 doi: 10.1097/MED.0000000000000476.

[4] Lenders JW, Eisenhofer G, Mannelli M, Pacak K. Phaeochromocytoma. Lancet. 2005;366:665-75 doi: 10.1016/S0140-6736(05)67139-5.

[5] Nolting S, Grossman A, Pacak K. Metastatic Phaeochromocytoma: Spinning Towards More Promising Treatment Options. Exp Clin Endocrinol Diabetes. 2018;127:117-28 doi: 10.1055/a-0715-1888.

[6] Baudin E, Habra MA, Deschamps F, Cote G, Dumont F, Cabanillas M, et al. Therapy of endocrine disease: treatment of malignant pheochromocytoma and paraganglioma. Eur J Endocrinol. 2014;171:R11122 doi: 10.1530/EJE-14-0113.

[7] Fishbein L, Leshchiner I, Walter V, Danilova L, Robertson AG, Johnson AR, et al. Comprehensive Molecular Characterization of Pheochromocytoma and Paraganglioma. Cancer Cell. 2017;31:181-93 doi: 10.1016/j.ccell.2017.01.001.

[8] Dahia PL, Ross KN, Wright ME, Hayashida CY, Santagata S, Barontini M, et al. A HIF1alpha regulatory loop links hypoxia and mitochondrial signals in pheochromocytomas. PLoS genetics. 2005;1:7280 doi: 10.1371/journal.pgen.0010008. 
reveals somatic mutations in pheochromocytoma and paraganglioma. Human molecular genetics. 2011;20:3974-85 doi: 10.1093/hmg/ddr324.

[10] Lussey-Lepoutre C, Buffet A, Gimenez-Roqueplo AP, Favier J. Mitochondrial Deficiencies in the Predisposition to Paraganglioma. Metabolites. 2017;7 doi: 10.3390/metabo7020017.

[11] Gimenez-Roqueplo AP, Dahia PL, Robledo M. An update on the genetics of paraganglioma, pheochromocytoma, and associated hereditary syndromes. Horm Metab Res. 2012;44:328-33 doi: 10.1055/s-0031-1301302.

[12] Crona J, Lamarca A, Ghosal S, Welin S, Skogseid B, Pacak K. Genotype-phenotype correlations in pheochromocytoma and paraganglioma. Endocr Relat Cancer. 2019 doi: 10.1530/ERC-19-0024.

[13] Amar L, Bertherat J, Baudin E, Ajzenberg C, Bressac-de Paillerets B, Chabre O, et al. Genetic testing in pheochromocytoma or functional paraganglioma. J Clin Oncol. 2005;23:8812-8 doi: 10.1200/JCO.2005.03.1484.

[14] Benn DE, Gimenez-Roqueplo AP, Reilly JR, Bertherat J, Burgess J, Byth K, et al. Clinical presentation and penetrance of pheochromocytoma/paraganglioma syndromes. J Clin Endocrinol Metab. 2006;91:827-36 doi: $10.1210 /$ jc. $2005-1862$.

[15] Letouze E, Martinelli C, Loriot C, Burnichon N, Abermil N, Ottolenghi C, et al. SDH Mutations Establish a Hypermethylator Phenotype in Paraganglioma. Cancer Cell. 2013;23:739-52 doi: 10.1016/j.ccr.2013.04.018.

[16] Cardaci S, Zheng L, MacKay G, van den Broek NJ, MacKenzie ED, Nixon C, et al. Pyruvate carboxylation enables growth of SDH-deficient cells by supporting aspartate biosynthesis. Nat Cell Biol. 2015;17:1317-26 doi: 10.1038/ncb3233.

[17] Sulkowski PL, Sundaram RK, Oeck S, Corso CD, Liu Y, Noorbakhsh S, et al. Krebs-cycle-deficient hereditary cancer syndromes are defined by defects in homologous-recombination DNA repair. Nat Genet. 2018;50:1086-92 doi: 10.1038/s41588-018-0170-4. 
Mitochondrial Electron Transport Chain in Cell Proliferation Is to Enable Aspartate Synthesis. Cell. 2015;162:540-51 doi: 10.1016/j.cell.2015.07.016.

[19] Cardaci S, Zheng L, MacKay G, Van den Broek NJF, MacKenzie ED, Nixon C, et al. Pyruvate carboxylation enables growth of SDH-deficient cells by supporting aspartate biosynthesis. Nat Cell Biol. 2015;17:1317 doi: 10.1038/ncb3233.

[20] Lussey-Lepoutre C, Hollinshead KER, Ludwig C, Menara M, Morin A, Castro-Vega LJ, et al. Loss of succinate dehydrogenase activity results in dependency on pyruvate carboxylation for cellular anabolism. Nat Commun. 2015;6 doi: ARTN 8784

10.1038/ncomms9784.

[21] Casero RA, Jr., Murray Stewart T, Pegg AE. Polyamine metabolism and cancer: treatments, challenges and opportunities. Nat Rev Cancer. 2018;18:681-95 doi: 10.1038/s41568-018-0050-3.

[22] Damiani E, Wallace HM. Polyamines and Cancer. Methods Mol Biol. 2018;1694:469-88 doi: 10.1007/978-1-4939-7398-9_39.

[23] Ekker M, Sourkes TL. Developmental pattern of ornithine decarboxylase activity, Sadenosylmethionine decarboxylase, and polyamines of rat adrenal glands. Biol Neonate. 1987;51:260-7 doi: $10.1159 / 000242662$.

[24] Schipper RG, Romain N, Otten AA, Tan J, Lange WP, Verhofstad AA. Immunocytochemical detection of ornithine decarboxylase. J Histochem Cytochem. 1999;47:1395-404 doi: 10.1177/002215549904701106. [25] Tonin PN, Yeger H, Stallings RL, Srinivasan PR, Lewis WH. Amplification of N-myc and ornithine decarboxylase genes in human neuroblastoma and hydroxyurea-resistant hamster cell lines. Oncogene. $1989 ; 4: 1117-21$.

[26] Maris JM, Hogarty MD, Bagatell R, Cohn SL. Neuroblastoma. Lancet. 2007;369:2106-20 doi: 10.1016/S0140-6736(07)60983-0.

[27] Simo M, Navarro X, Yuste VJ, Bruna J. Autonomic nervous system and cancer. Clin Auton Res. 2018;28:301-14 doi: 10.1007/s10286-018-0523-1. 
human neuroblastomas correlates with advanced disease stage. Science. 1984;224:1121-4 doi: 10.1126/science.6719137.

[29] Bachmann AS, Geerts D. Polyamine synthesis as a target of MYC oncogenes. J Biol Chem. 2018;293:18757-69 doi: 10.1074/jbc.TM118.003336.

[30] Bello-Fernandez C, Packham G, Cleveland JL. The ornithine decarboxylase gene is a transcriptional target of c-Myc. Proc Natl Acad Sci U S A. 1993;90:7804-8 doi: 10.1073/pnas.90.16.7804.

[31] Hogarty MD, Norris MD, Davis K, Liu X, Evageliou NF, Hayes CS, et al. ODC1 is a critical determinant of MYCN oncogenesis and a therapeutic target in neuroblastoma. Cancer Res. 2008;68:9735-45 doi: 10.1158/0008-5472.CAN-07-6866.

[32] Geerts D, Koster J, Albert D, Koomoa DL, Feith DJ, Pegg AE, et al. The polyamine metabolism genes ornithine decarboxylase and antizyme 2 predict aggressive behavior in neuroblastomas with and without MYCN amplification. Int J Cancer. 2010;126:2012-24 doi: 10.1002/ijc.25074.

[33] Rounbehler RJ, Li W, Hall MA, Yang C, Fallahi M, Cleveland JL. Targeting ornithine decarboxylase impairs development of MYCN-amplified neuroblastoma. Cancer Res. 2009;69:547-53 doi: 10.1158/00085472.CAN-08-2968.

[34] Valentijn LJ, Koster J, Haneveld F, Aissa RA, van Sluis P, Broekmans ME, et al. Functional MYCN signature predicts outcome of neuroblastoma irrespective of MYCN amplification. Proc Natl Acad Sci U S A. 2012;109:19190-5 doi: 10.1073/pnas.1208215109.

[35] Gamble LD, Purgato S, Murray J, Xiao L, Yu DMT, Hanssen KM, et al. Inhibition of polyamine synthesis and uptake reduces tumor progression and prolongs survival in mouse models of neuroblastoma. Sci Transl Med. 2019;11 doi: 10.1126/scitranslmed.aau1099.

[36] Evageliou NF, Haber M, Vu A, Laetsch TW, Murray J, Gamble LD, et al. Polyamine Antagonist Therapies Inhibit Neuroblastoma Initiation and Progression. Clin Cancer Res. 2016;22:4391-404 doi: 10.1158/1078-0432.CCR-15-2539. 
neuroblastoma. Clin Cancer Res. 2009;15:5956-61 doi: 10.1158/1078-0432.CCR-08-3213.

[38] Sarhan S, Knodgen B, Seiler N. Polyamine deprivation, malnutrition and tumor growth. Anticancer Res. 1992;12:457-66.

[39] Aziz SM, Gillespie MN, Crooks PA, Tofiq SF, Tsuboi CP, Olson JW, et al. The potential of a novel polyamine transport inhibitor in cancer chemotherapy. J Pharmacol Exp Ther. 1996;278:185-92.

[40] Porter CW, Cavanaugh PF, Jr., Stolowich N, Ganis B, Kelly E, Bergeron RJ. Biological properties of N4- and N1,N8-spermidine derivatives in cultured L1210 leukemia cells. Cancer Res. 1985;45:2050-7.

[41] Ghayee HK, Bhagwandin VJ, Stastny V, Click A, Ding LH, Mizrachi D, et al. Progenitor cell line (hPheo1) derived from a human pheochromocytoma tumor. PLoS One. 2013;8:e65624 doi: 10.1371/journal.pone.0065624.

[42] Lorenz MA, Burant CF, Kennedy RT. Reducing time and increasing sensitivity in sample preparation for adherent mammalian cell metabolomics. Anal Chem. 2011;83:3406-14 doi: 10.1021/ac103313x.

[43] Soga T, Heiger DN. Amino acid analysis by capillary electrophoresis electrospray ionization mass spectrometry. Anal Chem. 2000;72:1236-41.

[44] Soga T, Ohashi Y, Ueno Y, Naraoka H, Tomita M, Nishioka T. Quantitative metabolome analysis using capillary electrophoresis mass spectrometry. J Proteome Res. 2003;2:488-94 doi: 10.1021/pr034020m. [45] Sugimoto M, Wong DT, Hirayama A, Soga T, Tomita M. Capillary electrophoresis mass spectrometrybased saliva metabolomics identified oral, breast and pancreatic cancer-specific profiles. Metabolomics. 2010;6:78-95 doi: 10.1007/s11306-009-0178-y.

[46] Eisenhofer G, Prejbisz A, Peitzsch M, Pamporaki C, Masjkur J, Rogowski-Lehmann N, et al. Biochemical Diagnosis of Chromaffin Cell Tumors in Patients at High and Low Risk of Disease: Plasma versus Urinary Free or Deconjugated O-Methylated Catecholamine Metabolites. Clin Chem. 2018;64:164656 doi: 10.1373/clinchem.2018.291369. 
Metabolic and Lipidomic Sample Preparation Workflow for Suspension-Cultured Mammalian Cells using Jurkat T lymphocyte Cells. J Proteomics Bioinform. 2015;8:126-32 doi: 10.4172/jpb.1000360.

[48] Clasquin MF, Melamud E, Rabinowitz JD. LC-MS data processing with MAVEN: a metabolomic analysis and visualization engine. Curr Protoc Bioinformatics. 2012; Chapter 14:Unit14 1 doi: 10.1002/0471250953.bi1411s37.

[49] Adusumilli R, Mallick P. Data Conversion with ProteoWizard msConvert. Methods Mol Biol. 2017;1550:339-68 doi: 10.1007/978-1-4939-6747-6_23.

[50] Chawade A, Alexandersson E, Levander F. Normalyzer: a tool for rapid evaluation of normalization methods for omics data sets. J Proteome Res. 2014;13:3114-20 doi: 10.1021/pr401264n.

[51] http://bioconductor.org.

[52] McCarthy DJ, Chen YS, Smyth GK. Differential expression analysis of multifactor RNA-Seq experiments with respect to biological variation. Nucleic Acids Research. 2012;40:4288-97 doi: 10.1093/nar/gks042.

[53] https://polly.elucidata.io/ PI.

[54] Sergushichev AA, Loboda AA, Jha AK, Vincent EE, Driggers EM, Jones RG, et al. GAM: a webservice for integrated transcriptional and metabolic network analysis. Nucleic Acids Research. 2016;44:W194-W200 doi: 10.1093/nar/gkw266.

[55] Shannon P, Markiel A, Ozier O, Baliga NS, Wang JT, Ramage D, et al. Cytoscape: A software environment for integrated models of biomolecular interaction networks. Genome Research. 2003;13:2498504 doi: $10.1101 /$ gr. 1239303.

[56] Bergeron RJ, Weimar WR, Luchetta G, Streiff RR, Wiegand J, Perrin J, et al. Metabolism and pharmacokinetics of N1,N11-diethylnorspermine. Drug Metab Dispos. 1995;23:1117-25.

[57] Kuefer R, Hofer MD, Altug V, Zorn C, Genze F, Kunzi-Rapp K, et al. Sodium butyrate and tributyrin induce in vivo growth inhibition and apoptosis in human prostate cancer. Br J Cancer. 2004;90:535-41 doi: 10.1038/sj.bjc.6601510. 
[59] Bernacki RJ, Oberman EJ, Seweryniak KE, Atwood A, Bergeron RJ, Porter CW. Preclinical antitumor efficacy of the polyamine analogue N1, N11-diethylnorspermine administered by multiple injection or continuous infusion. Clin Cancer Res. 1995;1:847-57.

[60] Fagerberg L, Hallstrom BM, Oksvold P, Kampf C, Djureinovic D, Odeberg J, et al. Analysis of the human tissue-specific expression by genome-wide integration of transcriptomics and antibody-based proteomics. Mol Cell Proteomics. 2014;13:397-406 doi: 10.1074/mcp.M113.035600.

[61] Richter S, Gieldon L, Pang Y, Peitzsch M, Huynh T, Leton R, et al. Metabolome-guided genomics to identify pathogenic variants in isocitrate dehydrogenase, fumarate hydratase, and succinate dehydrogenase genes in pheochromocytoma and paraganglioma. Genet Med. 2019;21:705-17 doi: 10.1038/s41436-0180106-5.

[62] Bernacki RJ, Bergeron RJ, Porter CW. Antitumor activity of N,N'-bis(ethyl)spermine homologues against human MALME-3 melanoma xenografts. Cancer Res. 1992;52:2424-30.

[63] Imperiale A, Moussallieh FM, Sebag F, Brunaud L, Barlier A, Elbayed K, et al. A new specific succinate-glutamate metabolomic hallmark in SDHx-related paragangliomas. PLoS One. 2013;8:e80539 doi: 10.1371/journal.pone.0080539.

[64] Richter S, Peitzsch M, Rapizzi E, Lenders JW, Qin N, de Cubas AA, et al. Krebs Cycle Metabolite Profiling for Identification and Stratification of Pheochromocytomas/Paragangliomas due to Succinate Dehydrogenase Deficiency. Journal of Clinical Endocrinology \& Metabolism. 2014;99:3903-11 doi: $10.1210 /$ jc. $2014-2151$.

[65] Gieldon L, William D, Hackmann K, Jahn W, Jahn A, Wagner J, et al. Optimizing Genetic Workup in Pheochromocytoma and Paraganglioma by Integrating Diagnostic and Research Approaches. Cancers (Basel). 2019;11 doi: 10.3390/cancers11060809.

[66] Tevosian SG, Ghayee HK. Pheochromocytoma/Paraganglioma: A Poster Child for Cancer Metabolism. J Clin Endocrinol Metab. 2018;103:1779-89 doi: 10.1210/jc.2017-01991. 
Hypoxia on Catecholamine Biosynthesis in Absence or Presence of Hif2alpha in Pheochromocytoma Cells. Cancers (Basel). 2019;11 doi: 10.3390/cancers 11050594.

[68] Caron PC, Cote LJ, Kremzner LT. Putrescine, a source of gamma-aminobutyric acid in the adrenal gland of the rat. Biochem J. 1988;251:559-62 doi: 10.1042/bj2510559.

[69] Madsen KK, Larsson OM, Schousboe A. Regulation of excitation by GABA neurotransmission: focus on metabolism and transport. Results Probl Cell Differ. 2008;44:201-21 doi: 10.1007/400_2007_036.

[70] Tran L, Fitzpatrick C, Cohn SL, Pytel P. Composite tumor with pheochromocytoma and immature neuroblastoma: report of two cases with cytogenetic analysis and discussion of current terminology. Virchows Arch. 2017;471:553-7 doi: 10.1007/s00428-017-2225-9.

[71] Shida Y, Igawa T, Abe K, Hakariya T, Takehara K, Onita T, et al. Composite pheochromocytoma of the adrenal gland: a case series. BMC Res Notes. 2015;8:257 doi: 10.1186/s13104-015-1233-6.

[72] Moon SB. Childhood neuroblastoma masquerading as pheochromocytoma: case report. Int Med Case Rep J. 2016;9:65-7 doi: 10.2147/IMCRJ.S100479. 


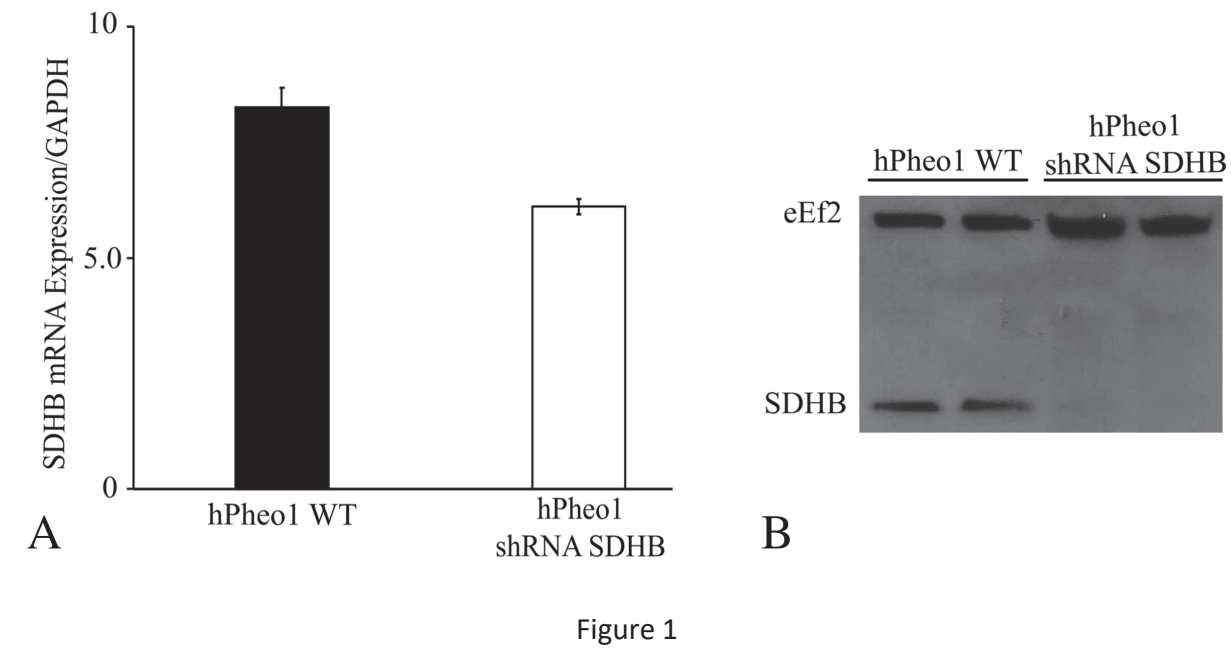


Cell Lines

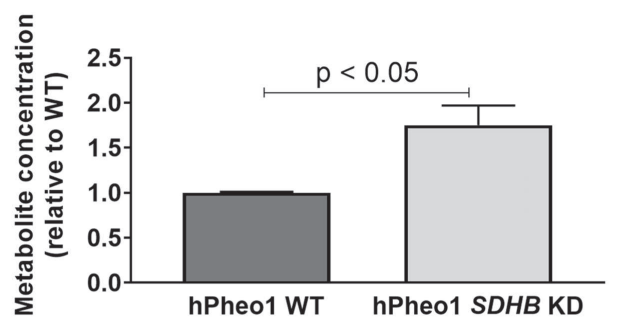

Tissue Samples

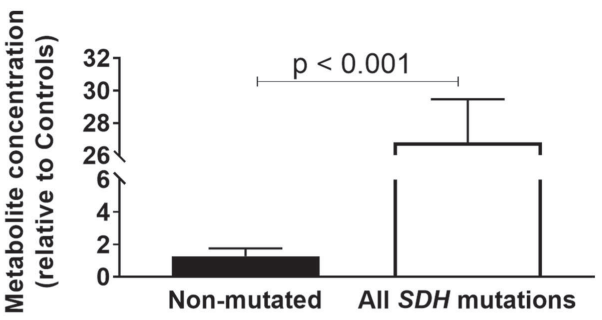

A) Succinate Levels

Arginine

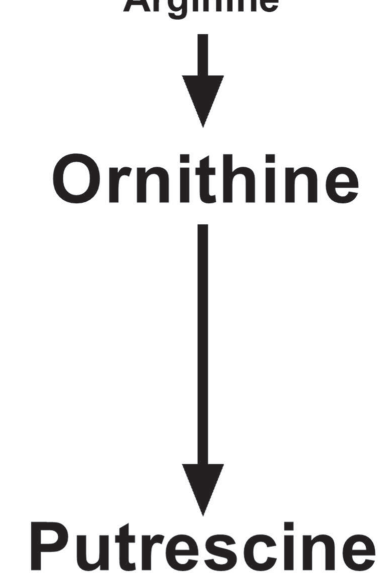

Spermidine
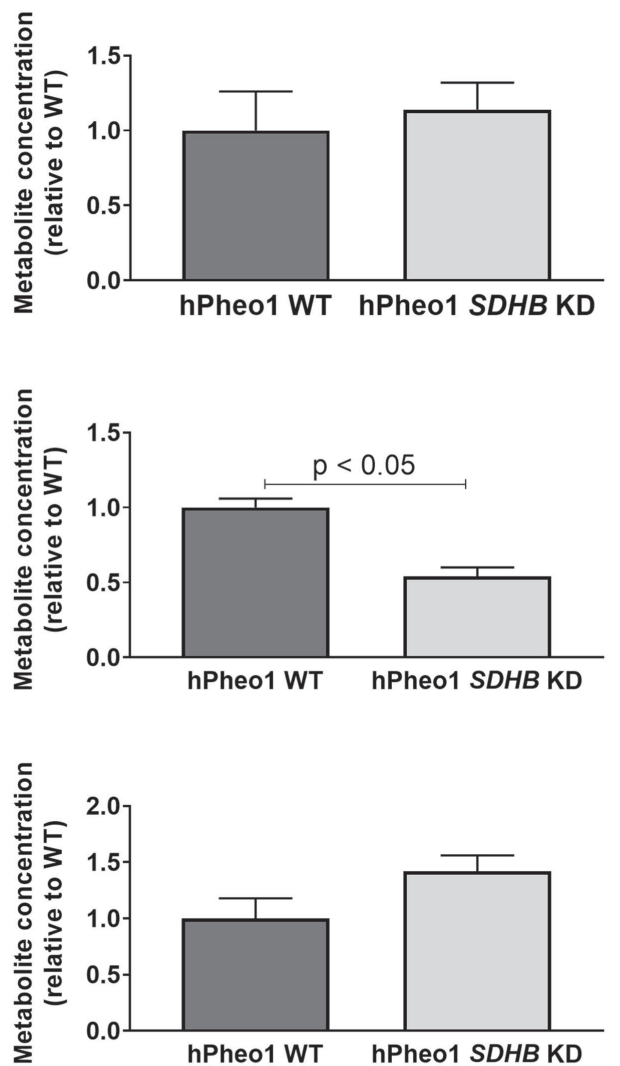

Spermine

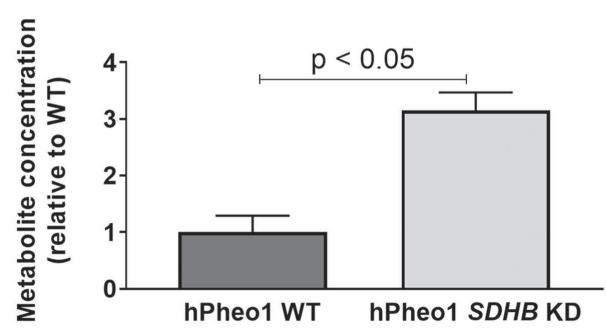

Tissue Samples
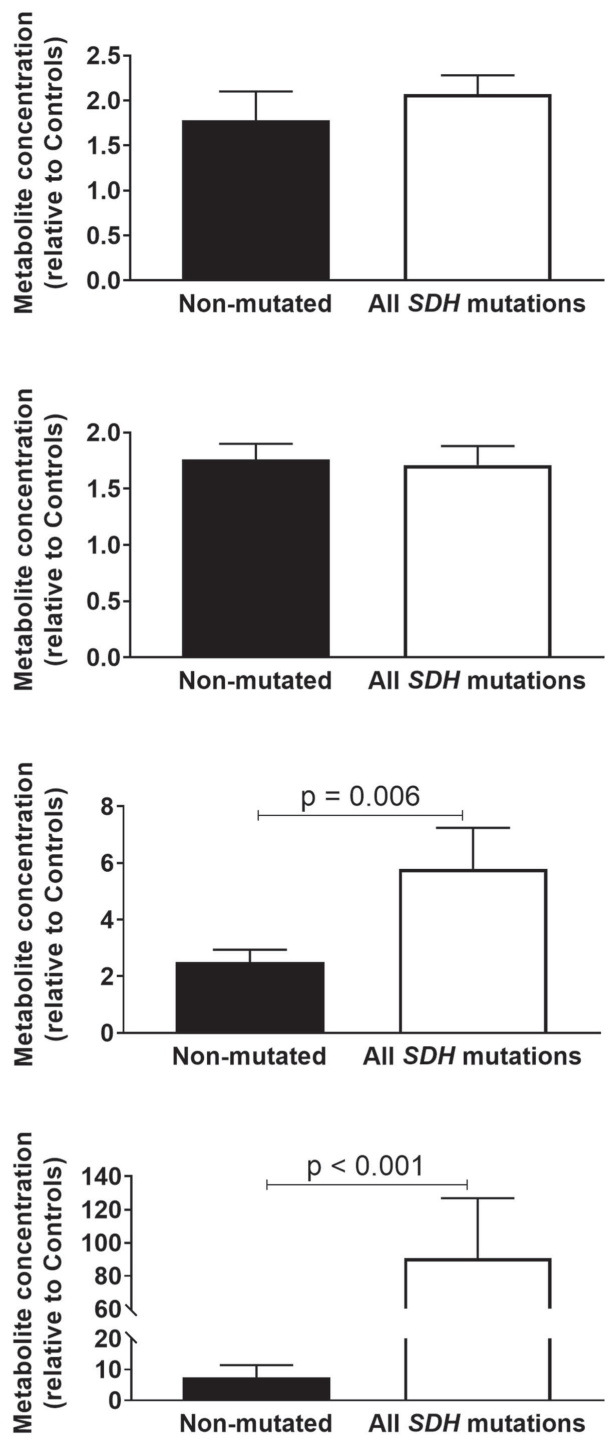

\section{B) Polyamine Pathway}


DENSPM dose ( $\mathrm{nM}$ )
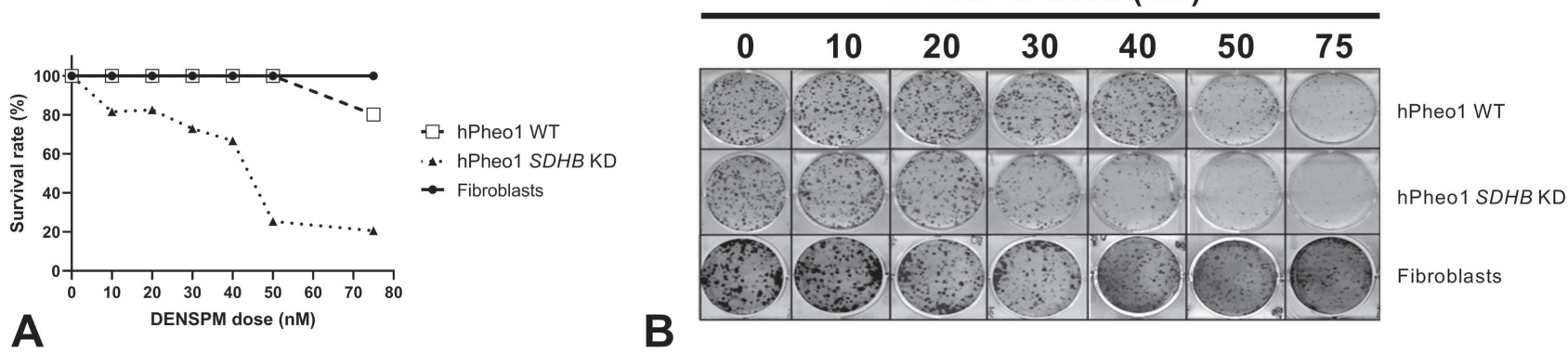

\section{A}

DENSPM dose (nM)

1. hPheo1 SDHB KD

- Fibroblasts

B

DESPM dose (nM)

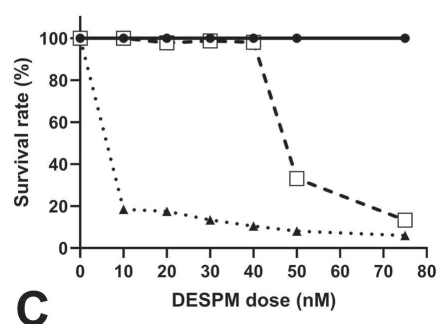

마 hPheo1 WT

1. hPheo1 SDHB KD

- Fibroblasts

D

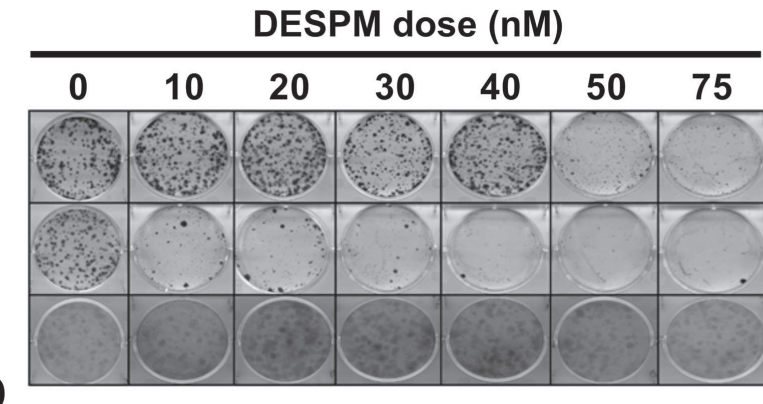

hPheo1 WT

hPheo1 SDHB KD

Figure 3 

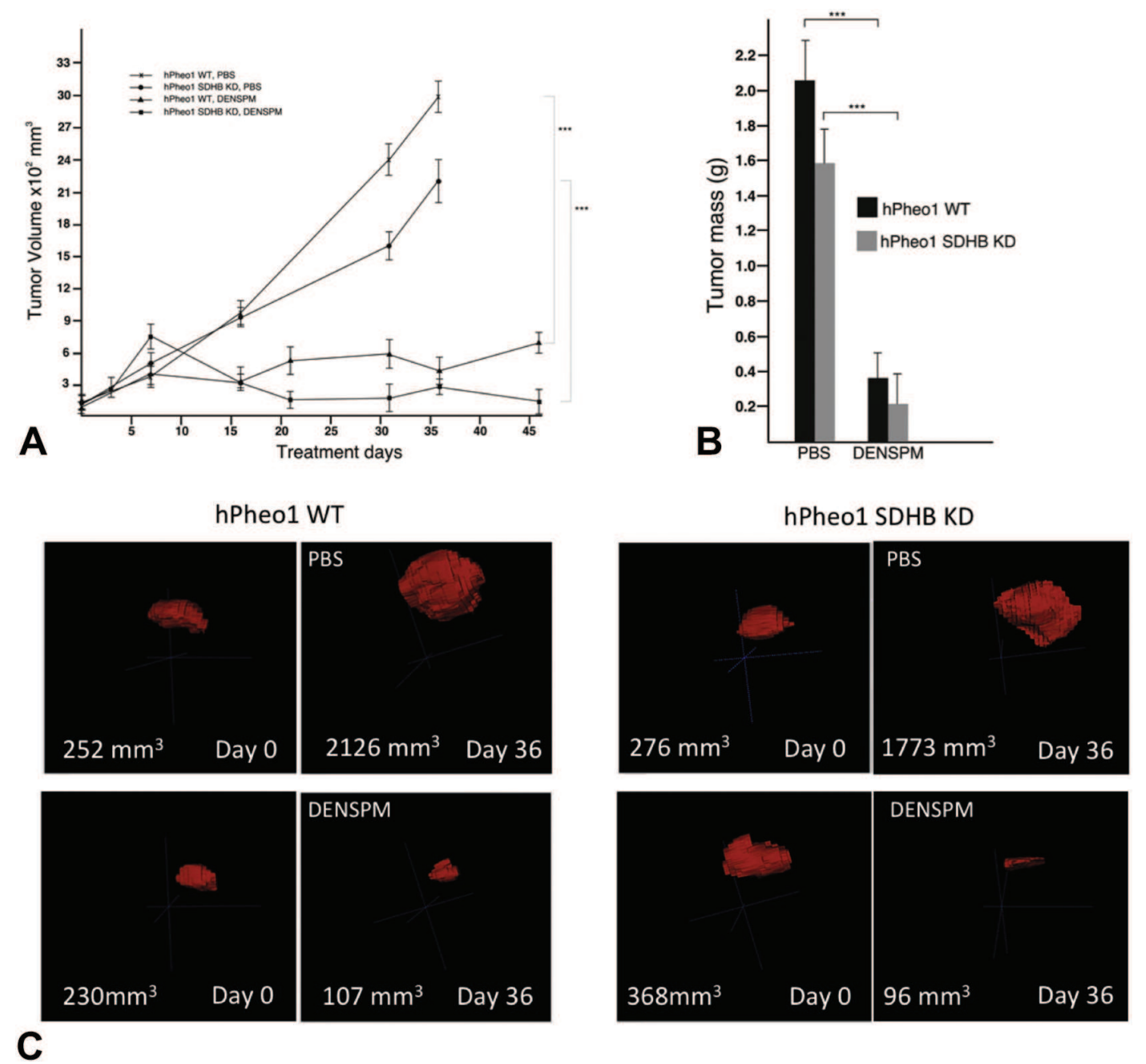

Figure 4 